\title{
Optimization for Smart Home Electricity Load Based on Real-time Pricing Environment
}

\author{
Tianqi Wu \\ School of electric power engineering, North China Electric Power University, Hebei Baoding 071000, \\ China.
}

547857718@qq.com

Keywords: Smart Home, Real-time Pricing, Optimization Control.

\begin{abstract}
In order to solve the smart home electricity load optimization problem, this paper firstly analyzes the situation of smart home optimization frame and estimates the smart home electricity load model. The electricity load optimization control model is established to achieve the aim of helping smart family to minimize the electricity consumption. Furthermore, the simulation results for the example in this paper verify the effectiveness and validity of the model.
\end{abstract}

\section{Introduction}

With the gradual deepening of the electricity reform, optimizing the allocation of grid resource and improving the operational efficiency of the electricity market are crucial for the reform of electricity industry. For the average home users, they prefer to concern about how to control household load and manage home energy to reduce the energy consumption and the cost of electricity according to the real-time pricing. And for the electricity market, realizing the target of peak load shifting in the implementation of demand response programs is one of the key problems in the future.

Therefore, specific to the smart home, to achieve electricity load optimization is very meaningful. Firstly we estimate the smart home electricity load model after the brief analysis. To realize the minimum of electricity cost, the electricity load optimization control model is established. And we set examples to verify the effectiveness and applicability of the model.

\section{Smart home electricity load model}

In real-time pricing programs, it is assumed that each user wants to optimize the energy structure in the next $n$ hours.Define A as electrical equipment set, which may include washing machine, dryer, refrigerator, hybrid car and so on. Each electrical equipment can be seen as a $\in$ A.The user needs to set up the demand for electricity in advance. So $e_{a}{ }^{n}$ refers to the electricity consumption of electrical equipment $a$ in the $n$ hour. We use $m_{a}$ to describe the starting time of electrical equipment $a$ and $n_{a}$ to describe the end time. We define $E_{a}$ as the energy consumption to run one time, which can be describe as the following formula:

$$
\sum_{m_{a}}^{n_{a}} e_{a}^{n}=E_{a}, \forall n \in\left[m_{a}, n_{a}\right]
$$

In general, each electrical appliance has a certain operating power in a limited range. The power constraint can be expressed as follow, which describes the upper and lower electricity consumption bounds of electrical equipment $a$.

$$
\beta_{\mathrm{a}}^{\min } / l \leq e_{a}^{n} \leq \beta_{\mathrm{a}}^{\max } / l, \forall n \in\left[m_{a}, n_{a}\right]
$$

The total electric power of each smart home has a certain upper limit, therefore there is a restriction for electricity consumption:

$$
\sum_{\mathrm{a} \in A} e_{a}^{n} \leq E_{\max } / l, \forall n \in L
$$

We can describe the general behavior of electrical equipment using the following formula set: 


$$
\Phi=\left\{\begin{array}{c}
\sum_{\mathrm{m}_{a}}^{n_{a}} e_{a}^{n}=E_{a}, \forall n \in\left[\mathrm{m}_{a}, n_{a}\right] \\
\beta_{a}^{\min } / l \leq e_{a}^{n} \leq \beta_{a}^{\max } / l, \forall n \in\left[\mathrm{m}_{a}, n_{a}\right] \\
\sum_{a \in A} e_{a}^{n} \leq E_{\max } / l, \forall n \in L \\
e_{a}^{n}=0, \forall n \notin\left[\mathrm{m}_{a}, n_{a}\right]
\end{array}\right.
$$

\section{The Electricity Load Optimization Control Model}

We assume that each user will install a smart meter with the function of two-way communication and real-time price forecast. Firstly, users only need to select the corresponding setting parameters of electrical equipment according to their own demand for electricity, such as $E_{a}, m_{a}, n_{a}$ and so on. According to the comprehensive power needs, scheduling module will choose the optimal electric power consumption vector for each device.

We set the target of the minimum electricity consumption as follow and estimate the electricity load optimization control model ${ }^{[1]}$.

$$
\begin{gathered}
\min \sum_{n=1}^{N} \operatorname{Pr} e(n) \times E_{n} \\
E_{n}=\sum_{n=1}^{N} e_{a}^{n}, a \in A \\
E_{a}=\sum_{n=m_{a}}^{n_{a}} e_{a}^{n}, \forall n \in\left[m_{a}, n_{a}\right] \\
\text { s.t. } \quad \beta_{a}^{\min } / l \leq e_{a}^{n} \leq \beta_{a}^{\max } / l \\
\sum_{a \in A} e_{a}^{n} \leq E_{\max } / l \\
e_{a}^{n}=y_{a}^{n} \times \beta_{a}^{\max } / l+\left(1-y_{a}^{n}\right) \times \beta_{a}^{\min } / l \\
y_{a}^{n}=0,1
\end{gathered}
$$

$\operatorname{Pre}(n)$ describes the electricity price of the $n$ th. hour. $E_{n}$ refers to the consumption in the $n$ th. hour. $y_{a}{ }^{n}$ is defined as a binary variable, which means electrical equipment is working when $y_{a}{ }^{n}=1$ and is opponent when $y_{a}{ }^{n}=0$.

\section{Analysis of Examples}

Considering a family with a variety of electrical equipment, it is assumed that the family will use real-time pricing programs. In order to express the problem in this article better, we select the following commonly used household equipment to create a task group: washing machines, water heaters, television, computers, lights, rice cookers, range hood, microwave oven, induction cooker, Yuba, hair dryers, irons, air conditioners, fans, water boilers, refrigerators, vacuum cleaners. The group includes not only the electrical equipment with fixed energy consumption, but also the relatively flexible electrical equipment that can be transferred from peak hours to off-peak hours.

The electricity price data ${ }^{[2]}$ to provide basis for decision optimization is collected from Fig.1. To increase the accuracy of data, we use the average of three years' prices.

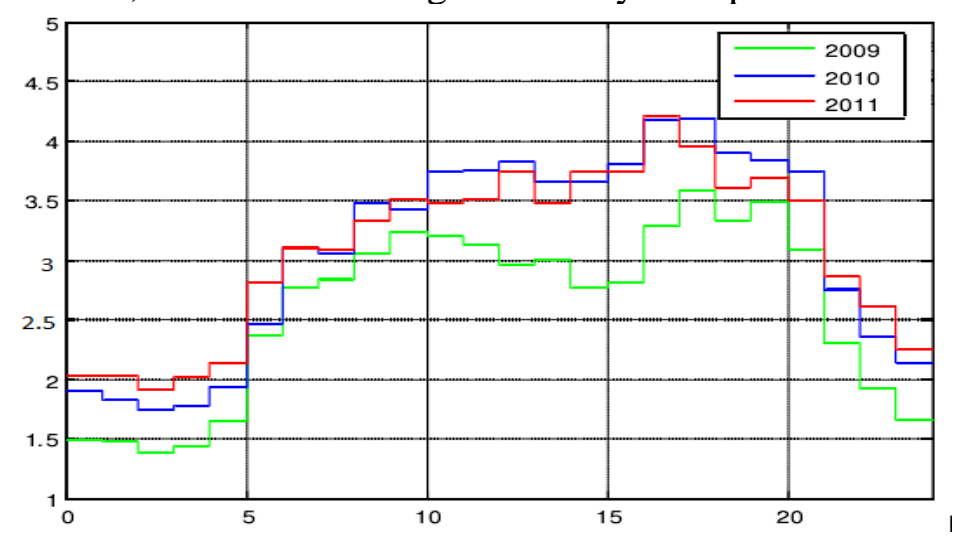

Fig.1 Average price curve in a day in different years 
The data of different equipment are collected from the literature and paper ${ }^{[3]}$. The data is about a family of three Canadians, and the family has three rooms and is totally 75 square meters. The main electricity consumption ${ }^{[4]}$ of different appliances in one day is shown as Table 1.

Table 1 The consumption of different appliances in a day

\begin{tabular}{cc} 
Appliances & Proportion \\
\hline refrigeration equipment & $24.6 \%$ \\
washer & $7 \%$ \\
dish-washing machine & $8.8 \%$ \\
cooking equipment & $15.8 \%$ \\
entertainment & $17.5 \%$ \\
illumination & $26.3 \%$ \\
\hline
\end{tabular}

Based on the model in the formula 5 above, we use Lingo to program and solve the problem according to the data of average electricity price curve in a day and the consumption of different appliances. Therefore, we can get the electricity cost curve in a day after the program of optimization model, which is shown as Fig. 2 comparing with the costs curve data before optimization.

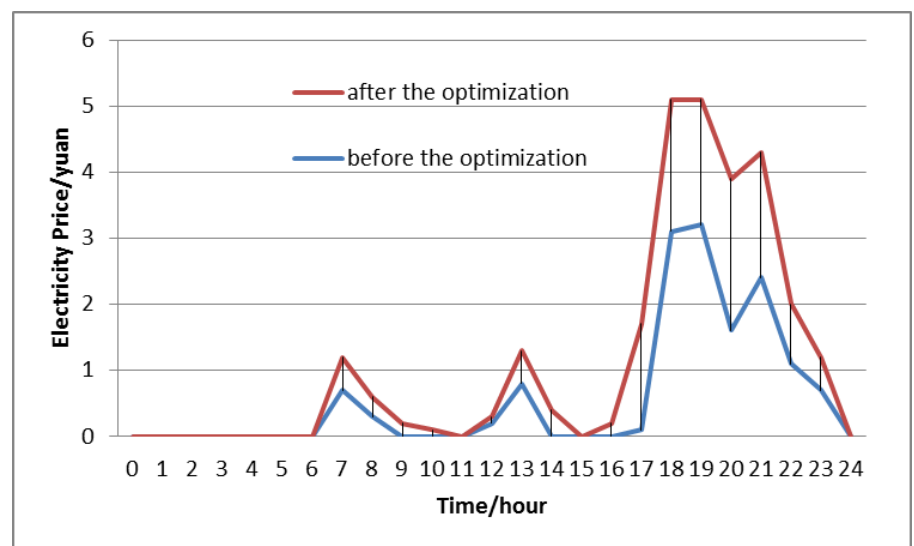

Fig.2 Comparison of electricity costs before and after optimization

\section{Summary}

From the Fig.2 we can know that there is a significant change for adjusting the peaking of electricity curve, aiming at reducing electricity consumption. Optimization results show that based on the real-time pricing environment, day electricity consumption curve becomes smoother and the peak of the electricity consumption curve was decreased significantly. Not only is the power system reliability improved greatly, but also the energy saving purpose is achieved. Therefore, it is beneficial for the power company and power supply system. For the home users, they are more concerned about whether they can reduce electricity costs or not. And we can learn from the figure obviously that the costs have been significantly reduced after the optimization. What's more, the results further verify the effectiveness and validity of the model. 


\section{References}

[1] Weipo Wu. Optimization for Family Energy Consumption in Real-time Pricing Environment [D]. Shanghai: Shanghai Jiaotong University, 2013.

[2] Baohi Liu. Research on Household Electricity Load Control Strategy Based on Demand Response [D]. Harbin: Harbin University of Science and Technology, 2014.

[3] Beausoleil-Morrison, An Experimental and Simulation Based Investigation of the Performance of Small-scale Fuel Cell and Combustion-based Cogeneration Devices Serving Residential Buildings [M/OL], 2008. http://www.ecbcs.org/docs/Annex 42 Final Report.pdf.

[4] Wei Yi. Research of Smart Home Energy Management Based on Demand Response [D]. Qinhuangdao: Yanshan University, 2015. 